\title{
Collaboration between doctors and pharmacists in the community
}

\author{
Debbie Rigby, Consultant Clinical Pharmacist, DR Pharmacy Consulting, Camp Hill, \\ Queensland
}

\begin{abstract}
Summary
The role of pharmacists is expanding in primary care. There is evidence that greater collaboration between general practitioners and pharmacists can improve patient care. Medication reviews are an example of how pharmacists can assist general practitioners. Joint training and co-location of practices should encourage increased collaboration between the professions. Key words: drug utilisation, medication reviews.
\end{abstract}

(Aust Prescr 2010;33:191-3)

\section{Introduction}

Teamwork, communication and collaboration between health professionals are important for the safe and effective delivery of health care. ${ }^{1}$ Australia's ageing population and the increasing burden of chronic disease present opportunities and imperatives for health professionals to practise collaboratively.

A literature review by the National Prescribing Service has identified significant problems associated with medication misadventure. Approximately $6 \%$ of hospital admissions are associated with adverse drug events and high error rates during transfer of care. Poor communication was the most important common factor contributing to medication errors. ${ }^{2}$ Increased interprofessional collaboration between doctors and pharmacists could therefore reduce the considerable medication-related morbidity and mortality.

\section{Role of pharmacists}

Pharmacy practice in Australia now involves patient-centred care including counselling, providing drug information, monitoring drug therapy and patient adherence, as well as the supply of medicines. Over the last decade, the role of pharmacists in the community has expanded with the provision of many professional services including medication reviews, diabetes and asthma management programs, and patient medication profiles.

It is in the additional role of managing medication therapy, in collaboration with prescribers, that pharmacists can now make a vital contribution to patient care. To do so, the role of the pharmacist needs to be redefined and reorientated. The traditional relationship between the doctor as prescriber, and pharmacist as dispenser, is no longer appropriate to ensure safety, effectiveness and adherence to therapy. Pharmacists need to pay more attention to patient-centred, outcomesfocused care to optimise the safe and effective use of medicines. Dispensing is, and must remain, a responsibility of the pharmacy profession, but prescribing and dispensing should not be done by the same person. By taking direct responsibility for individual patients' medication-related needs, pharmacists can make a unique contribution to the outcome of medication therapy and to their patients' quality of life. ${ }^{3}$

\section{Collaborative practice}

Australian and international studies have shown the benefits pharmacists can make to direct patient care and better medication management. ${ }^{4,5}$ In the UK and New Zealand, reviews of medicine use have contributed to professional integration and patient care. 6,7 In Canada, early concerns about collaborative practice have been resolved as general practitioners discovered the benefits of working with pharmacists. ${ }^{89}$ General practitioners are more likely to accept a pharmacist's recommendations if they have personal contact in case conferences than they are if they are sent written recommendations. ${ }^{10}$ General practitioners may be reluctant to use a service led by a pharmacist who they do not personally know. ${ }^{11}$

The TEAMCare coordinated care trial demonstrated that pharmacists and general practitioners can work together in a primary care environment, although a greater degree of trust and collaboration is required. ${ }^{12}$ Trust appears to grow over time. When pharmacists are co-located with general practitioners there is a greater opportunity for trust to develop. ${ }^{13}$ However, the full effect of pharmacist integration may take longer than one year to perceive clearly. ${ }^{14}$

Studies that have integrated pharmacists into primary care practices have shown improved patient outcomes. ${ }^{15}$ Collaborative models have improved the treatment of hypertension. ${ }^{16}$ Pharmacists have the potential to optimise drug therapy by identifying medication-therapy problems and recommending solutions. ${ }^{17}$ Prescribers are receptive to such recommendations. ${ }^{18}$ Pharmacist-patient consultations in relation to medication management within general practitioners' surgeries and in patients' homes have high acceptability to patients. ${ }^{19}$

A role for a pharmacist within a general practice has been proposed to provide multiple risk management strategies 
to improve medication safety. The role would focus on interventions to high-risk patient groups and disease states, and would use practice information technology systems to detect potential safety problems. ${ }^{20}$

Interdisciplinary teaching of pharmacotherapeutics provides health professionals with greater insight into their respective roles. This could improve the quality use of medicines and reduce medication errors. ${ }^{21}$

\section{Medication reviews}

Medication reviews show the benefits of cooperation. Government remuneration for medication reviews by pharmacists began in 1997 in residential aged care facilities and in $\mathbf{2 0 0 1}$ for community patients. Collaborative medication reviews are included in many general practitioner and pharmacist practices, clinical practice guidelines and decision support tools. Several randomised trials have shown improvements in prescribing, and reduced healthcare use and medication costs following medication reviews in patients with hypertension, hyperlipidaemia and diabetes. ${ }^{22-24}$

The evidence supporting the benefits of home medicines reviews continues to expand. They can be effective in delaying the time to next hospitalisation for heart failure, ${ }^{25}$ identifying drug-related problems among people receiving treatment for mental illnesses, ${ }^{26}$ and assisting in the resolution of medication-related problems. ${ }^{27}$ Medication reviews after discharge from hospital have reduced morbidity and mortality in patients with heart failure. ${ }^{28}$

Despite this evidence and considerable support by the Pharmacy Guild and Divisions of General Practice, home medicine reviews are still underused. For example, they are not used enough in the detection and prevention of medication-related problems in cardiovascular disease. ${ }^{29}$

\section{Challenges to collaboration}

The dichotomous nature of community pharmacy practice is a critical dilemma for the profession. The role of community pharmacists has been traditionally characterised by dispensing prescription medicines, selling over-the-counter medication and offering healthcare advice. Community pharmacists are often not viewed as a core part of the primary healthcare team. Perceptions around being a retailer and healthcare provider create uncertainty in the minds of the medical profession, funders and consumers. Pharmacy is the only health profession that is reimbursed for its sale of a product rather than provision of a service.

Currently community pharmacists have limited opportunity to see patients in a primary care setting as part of a multidisciplinary team. Direct contact between community pharmacists and general practitioners is often brief and can be perceived as adversarial.

In many cases geographical isolation and separate premises are barriers to the integration of community pharmacists into the primary healthcare team. Electronic health records will potentially overcome some of the barriers with shared access to medication profiles and secure transfer of information. Lack of a private consultation area in a community pharmacy is also a barrier. In addition, the attitudes of doctors towards pharmacists and their contribution to better medication management is another barrier to overcome. ${ }^{13}$

Some medical organisations have been critical of an expanded role for pharmacists in primary health care, opposing pharmacy as the first point of call for treating minor ailments, pharmacist prescribing, disease state management, immunisation and sick notes. However, pharmacists already play a valuable role in triaging minor conditions in the community. People will continue to consult pharmacists for minor health problems as they are a trusted and accessible source of information and advice.

\section{Conclusion}

The roles of the doctor and pharmacist are complementary. Good working relationships between all healthcare professionals are essential to the delivery of personalised and effective patient services. All health professions must show greater responsiveness to changing patient needs.

Pharmacists have the skills and knowledge to contribute to the quality use of medicines, to minimise medication misadventure and to help consumers better manage their medicines. Interdisciplinary clinical teaching, communication and relationships are the keys to improving collaboration to achieve optimal medication management. Interprofessional collaboration between general practitioners and pharmacists must continue to evolve to meet the medication management and healthcare needs of the community now and in the future.

\section{References}

The full list of references is available online at www.australianprescriber.com with this article in Vol 33 No 6.

1. Institute of Medicine. Creating safety systems in health care organizations. In: Kohn L, Corrigan J, Donaldson M, eds. To err is human: building a safer health system. Washington (DC): National Academy Press; 2001.

12. McDonald $P$, Rigby $D$, Roush $P$, Watts $I$. GP referral in a group of older community-dwelling people. Aust Pharm 2000;19:107-11.

13. Bradley F, Elvey R, Ashcroft DM, Hassell K, Kendall J, Sibbald $B$, et al. The challenge of integrating community pharmacists into the primary health care team: a case study of local pharmaceutical services (LPS) pilots and interprofessional collaboration. J Interprof Care 2008;22:387-98.

15. Dolovich L, Kaczorowski J, Howard M, Rodriguez MC, Gaebel K, Haq M, et al; IMPACT team. Cardiovascular outcomes of a pharmaceutical care program integrated into family practices [abstract 21]. Can J Clin Pharmacol 2007;14:e116.

20. Ackerman E, Williams ID, Freeman C. Pharmacists in general practice - a proposed role in the multidisciplinary team. Aust Fam Physician 2010;39:163-4. 
25. Roughead EE, Barratt JD, Ramsay E, Pratt N, Ryan P, Peck R, et al. The effectiveness of collaborative medicine reviews in delaying time to next hospitalization for patients with heart failure in the practice setting. Circ Heart Fail 2009;2:424-8.

27. Gilbert AL, Roughead EE, Beilby J, Mott K, Barratt JD.

Collaborative medication management services: improving patient care. Med J Aust 2002;177:189-92.
28. Ponniah A, Anderson B, Shakib S, Doecke CJ, Angley M. Pharmacists' role in the post-discharge management of patients with heart failure: a literature review. J Clin Pharm Ther 2007;32:343-52.

Conflict of interest: none declared

\section{New drugs}

Some of the views expressed in the following notes on newly approved products should be regarded as tentative, as there may be limited published data and little experience in Australia of their safety or efficacy. However, the Editorial Executive Committee believes that comments made in good faith at an early stage may still be of value. As a result of fuller experience, initial comments may need to be modified. The Committee is prepared to do this. Before new drugs are prescribed, the Committee believes it is important that full information is obtained either from the manufacturer's approved product information, a drug information centre or some other appropriate source.

\section{Degarelix}

Firmagon (Ferring)

vials containing $80 \mathrm{mg}$ and $120 \mathrm{mg}$ as powder for reconstitution

Approved indication: prostate cancer

Australian Medicines Handbook section 14.3

Androgen deprivation is one approach to the treatment of prostate cancer. This can be achieved by using agonists of gonadotrophin releasing hormone such as goserelin and leuprorelin. Although these drugs cause an initial surge in testosterone, long-term use leads to decreased production.

Degarelix reduces testosterone production by antagonising gonadotrophin releasing hormone. By blocking the pituitary receptors, degarelix cuts testosterone concentrations within a few days, without the surge seen with gonadotrophin releasing hormone agonists.

In a dose-ranging study, 127 patients were randomised to take a starting dose of degarelix followed by monthly maintenance doses. Within three days the testosterone concentration had fallen into the target range in $89 \%$ of the men. Low levels were maintained in most of the 87 men who completed the one-year study. Prostate specific antigen was also reduced. ${ }^{1}$

Degarelix has to be given by subcutaneous injection into the abdomen. A depot is thought to form at the injection site so that the drug is slowly released. The half-life of the maintenance dose is estimated to be 28 days. Most of the dose is metabolised by hydrolysis and excreted in the faeces. The dose does not have to be adjusted in patients with mild to moderate renal or hepatic impairment.

Degarelix has been compared with intramuscular leuprorelin in a 12-month study. The 610 men in the study had prostate cancers ranging from localised to metastatic. Those who were randomised to take degarelix were given $240 \mathrm{mg}$ followed by monthly maintenance doses of $80 \mathrm{mg}$ or $160 \mathrm{mg}$. The desired testosterone concentration was achieved by $97-98 \%$ of the degarelix groups and $96 \%$ of the leuprorelin group.
The reduction in testosterone was more rapid in the degarelix groups. A similar pattern was seen with the reduction in prostate specific antigen. ${ }^{2}$

Adverse effects are common with degarelix. In the comparative study, $40 \%$ of patients had injection-site reactions with degarelix. Less than $1 \%$ of the leuprorelin group had injection-site reactions. Other adverse effects reported in the trial included flushing, weight gain and altered liver function. Adverse events resulted in approximately $7-9 \%$ of the degarelix group and $6 \%$ of the leuprorelin group discontinuing treatment. ${ }^{2}$ During treatment with degarelix the QTC interval on the ECG can be prolonged and some patients will develop anaemia. Some patients develop antibodies to degarelix although it is yet unclear whether this affects long-term efficacy. Although androgen deprivation has metabolic effects, lipids other than cholesterol, and glucose were not studied. Hypercholesterolaemia occurred in $5 \%$ of patients given degarelix. ${ }^{2}$

It appears that an antagonist of gonadotrophin releasing hormone is as effective as an agonist in reducing testosterone concentrations. While at first the reduction is more rapid than with leuprorelin, after about a month there is no significant difference between treatments. Further study will be needed to see the effect of degarelix on survival and whether it has any role in patients who have not responded to a gonadotrophin releasing hormone agonist.

$\begin{array}{lll}\mathbf{T} & \mathbf{T} \mathbf{T} \text { manufacturer provided clinical evaluation }\end{array}$

\section{References ${ }^{*}+\mathrm{A}$}

1. Gittelman M, Pommerville PJ, Persson BE, Jensen JK, Olesen TK; Degarelix Study Group. A 1-year, open label, randomized phase II dose finding study of degarelix for the treatment of prostate cancer in North America. J Urol 2008;180:1986-92.

2. Klotz L, Boccon-Gibod L, Shore ND, Andreou C, Persson BE, Cantor $\mathrm{P}$, et al. The efficacy and safety of degarelix: a 12-month, comparative, randomized, open-label, parallelgroup phase III study in patients with prostate cancer. BJU Int 2008;102:1531-8. 